\title{
A Mechanism for Savings in the Cerebellum
}

\author{
Javier F. Medina, Keith S. Garcia, and Michael D. Mauk \\ W. M. Keck Center for the Neurobiology of Learning and Memory, and Department of Neurobiology and Anatomy, \\ University of Texas Medical School, Houston, Texas 77030
}

The phenomenon of savings (the ability to relearn faster than the first time) is a familiar property of many learning systems. The utility of savings makes its underlying mechanisms of special interest. We used a combination of computer simulations and reversible lesions to investigate mechanisms of savings that operate in the cerebellum during eyelid conditioning, a well characterized form of motor learning. The results suggest that a site of plasticity outside the cerebellar cortex (possibly in the cerebellar nucleus) can be protected from the full consequences of extinction and that the residual plasticity that remains can later contribute to the savings seen during relearning.

Key words: plasticity; learning; memory; eyelid conditioning; simulation; LTD; LTP; extinction
Riding a bicycle, playing the piano, and typing are examples of motor skills that illustrate our capacity for relearning that is much more rapid than the original learning. In the laboratory, this rapid relearning is known as savings and has been frequently studied in the context of Pavlovian conditioning of motor responses. During conditioning of eyelid responses for example, paired presentations of a tone [the conditioned stimulus (CS)] and a reinforcing unconditioned stimulus (US), such as periorbital electrical stimulation, promote the acquisition of a conditioned motor response (closing the eyelid in response to the tone). These conditioned responses can be unlearned during extinction training in which the CS is not paired with the US (Schneiderman et al., 1962). During reacquisition training, savings is apparent because relearning occurs much faster than original learning (Frey and Ross, 1968; Napier et al., 1992). The existence of savings is among the evidence supporting the notion that extinction training leaves behind residual excitatory strength (Reberg, 1972; Schactman et al., 1985; Kehoe, 1988). Here we present evidence for this residual-plasticity hypothesis in eyelid conditioning and characterize basic mechanisms involved in the savings observed with this form of motor learning.

A variety of evidence indicates that the cerebellum is a necessary component of the neural pathways that mediate eyelid conditioning (Thompson, 1986; Thompson and Krupa, 1994; Raymond et al., 1996; Kim and Thompson, 1997). This evidence, combined with the well characterized synaptic organization and physiology of the cerebellum (Eccles et al., 1967; Llinas, 1981; Ito, 1984; Voogd and Glickstein, 1998), makes it possible to build large-scale computer simulations of the cerebellum and to test their capacity to display eyelid conditioning. We have shown previously that, by incorporating the evidence for plasticity at two sites (one in the cerebellar cortex and one in the cerebellar interpositus nucleus), these simulations can emulate the acquisition and extinction of conditioned responses (Medina et al.,

Received Jan. 26, 2001; revised March 16, 2001; accepted March 19, 2001.

This work was supported by National Institutes of Health Grants MH57051 and MH46904.

Correspondence should be addressed to Michael D. Mauk, Department of Neurobiology and Anatomy, University of Texas Medical School, 6431 Fannin, Houston, TX 77030. E-mail:m.mauk@uth.tmc.edu.

Copyright (c) 2001 Society for Neuroscience 0270-6474/01/214081-09\$15.00/0
2000). Here we report that these same simulations also display savings, even after extensive extinction training. In the simulations, the rules for plasticity combine with network properties in a way that makes rapid reversal of acquisition-related plasticity in the cerebellar cortex primarily responsible for extinction but keeps plasticity in the cerebellar nucleus relatively resistant to extinction training. Thus, the simulations suggest that persistent plasticity in the cerebellar nucleus is a form of residual memory that can contribute to savings.

To test this prediction, we used a reversible blockade technique to assess the presence of residual plasticity at various stages during extinction. As reported previously (Garcia and Mauk, 1998), infusion of picrotoxin into the interpositus nucleus functionally disconnects the cerebellar cortex and unmasks short-latency conditioned responses. Although other possibilities remain (see Discussion), recent evidence suggests that these short-latency responses are mediated by plasticity in the interpositus nucleus (Garcia and Mauk, 1998; Nores et al., 1999; Steele et al., 1999). In this study, we find that, although normal conditioned responses disappear during the first day of extinction training, residual plasticity, as revealed by short-latency responding after picrotoxin, persists even after $45 \mathrm{~d}$ of extinction training. We also observe that the rate of reacquisition for individual rabbits correlates with the magnitude of the short-latency responses unmasked by picrotoxin infusions on the last day of extinction before reacquisition. These findings suggest that the plasticity mediating the short-latency responses remains after extinction training and is at least partly responsible for the savings observed during reacquisition.

\section{MATERIALS AND METHODS}

Computer simulations. As described previously (Medina et al., 2000), the construction of the simulation was designed to represent the well characterized synaptic organization and physiology of the cerebellum (Fig. 1) (Eccles et al., 1967; Llinas, 1981; Ito, 1984; Voogd and Glickstein, 1998). For each neuron, we used a single-compartment, integrate-and-fire representation. This implementation solves for membrane potential based on leak and synaptic conductances. The main simplification is that a spike occurs when membrane potential exceeds a threshold value, which itself varies according to previous activity to allow for absolute and relative refractory periods. With the exception of a few unknown values, the cellular and synaptic properties were based on published reports (Medina et al., 2000). As reported previously, the results did not depend on the particular values of the small number of free parameters (Medina 


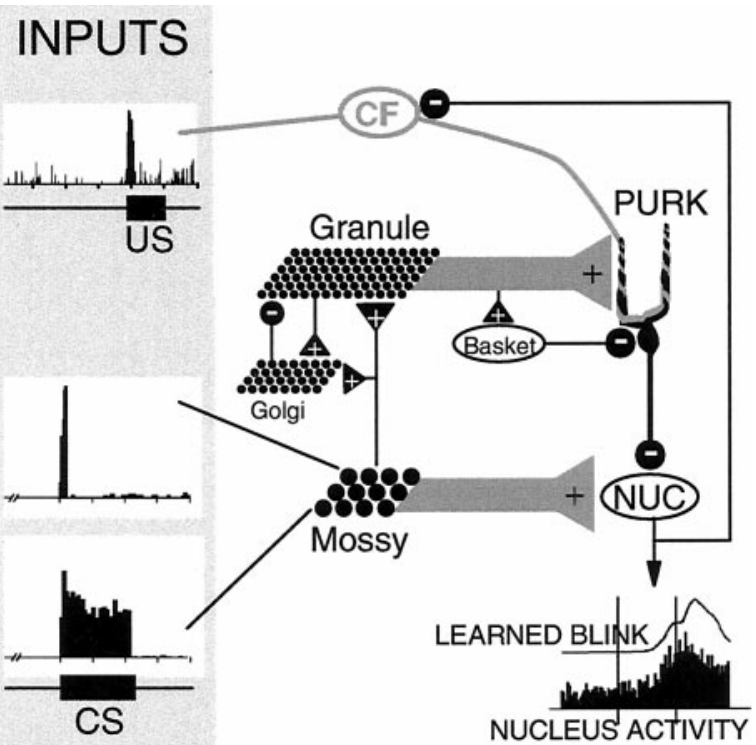

Figure 1. The relationship between eyelid conditioning and the circuitry of the cerebellum. During eyelid conditioning, mossy fibers (Mossy) convey information about the CS. This afferent influences cerebellar nucleus output through direct excitatory connections onto the nucleus cells ( $m f \rightarrow n u c$ synapses) and through a more indirect projection onto a large number of granule cells ( $g r \rightarrow P k j$ synapses; Granule) that ultimately results in Purkinje (PURK) cell inhibition of nucleus cells (NUC). This indirect pathway in the cerebellar cortex also involves two other types of inhibitory interneurons: basket cells (Basket), which inhibit Purkinje cells, and Golgi cells (Golgi), which inhibit granule cells and are in turn excited by both mossy fibers and granule cells. Climbing fibers $(C F)$, which make strong excitatory connections onto the Purkinje cells, have been shown to convey information about the US. Finally, increases in nucleus cell activity in response to the CS are known to drive the expression of conditioned responses. In the simulations, this learning is driven by changes in the strength of both $m f \rightarrow n u c$ and $g r \rightarrow P k j$ synapses.

et al., 2000, their Table 1). Interconnections between simulated neurons were based on the known numeric ratios of cells, divergence and convergence ratios of connections, and geometry of projections (Fig. 1). Maintaining these ratios as accurately as possible requires extremely large number of synapses $(>250,000)$, making the simulation very computationally intensive (it takes $\sim 10$ processor hours in our Alpha 500 workstation to simulate a $1 \mathrm{hr}$ conditioning session). Two sets of synapses in the simulation were modifiable, as suggested by empirical evidence (Robinson, 1976; Perrett et al., 1993; Raymond et al., 1996; Mauk, 1997). Climbing fiber inputs controlled activity-dependent plasticity at $g r \rightarrow P k j$ synapses. Long-term depression (LTD) was induced in $g r \rightarrow P k j$ synapses that were active at the time of a climbing fiber input (Sakurai, 1987; Ito, 1989; Hirano, 1990; Linden, 1994), whereas long-term potentiation (LTP) was induced in $g r \rightarrow P k j$ synapses that were active in the absence of a climbing fiber input (Sakurai, 1987; Hirano, 1990; Shibuki and Okada, 1992; Salin et al., 1996). The precise interval of time between granule cell and climbing fiber activation that is required for induction of LTD remains under investigation. Reported effective intervals have ranged from presynaptic first by $250 \mathrm{msec}$ (Chen and Thompson, 1995) to climbing fiber first by $125 \mathrm{msec}$ (Ekerot and Kano, 1989). Because we find that the results do not differ significantly for this range of intervals, our choice of a $0 \mathrm{msec}$ interval (i.e., simultaneous activity in $g r \rightarrow P k j$ synapse and climbing fiber input) represents a consensus interval that allows induction of LTD under a variety of experimental conditions (Ekerot and Kano, 1989; Karachot et al., 1994; Chen and Thompson, 1995). The reversibility of plasticity at $g r \rightarrow P k j$ synapses is at present an assumption of the simulation because the evidence to date suggests that LTP-LTD at this synapse are not mutually reversible because the expression of the former seems to be presynaptic (Salin et al., 1996), whereas that of the later is clearly postsynaptic (Linden, 1994). As first suggested by Miles and Lisberger (1981) and supported more recently by theoretical (Medina and Mauk, 1999) and empirical (Perrett and Mauk, 1995; Aizenman and Linden, 2000) analyses, plasticity in the cerebellar nucleus was controlled by Purkinje cell inputs. Thus, the excitatory $m f \rightarrow n u c$ synapses underwent LTD when active during strong inhibitory input from the Purkinje cells and LTP when active during a transient pause in this inhibition. Instead of this form of synaptic plasticity at $m f \rightarrow n u c$ synapses, pilot simulations incorporated the recently reported form of nonsynaptic plasticity that involves changes in nucleus cell excitability (Aizenman and Linden, 2000). The results presented here did not depend on whether synaptic or nonsynaptic plasticity was incorporated in the cerebellar nucleus, as long as this plasticity was under the control of Purkinje cell inputs.

The way in which this computer representation of the cerebellum is engaged during simulated eyelid conditioning has been presented previously (Medina et al., 2000) and will be described only briefly. Simulating eyelid conditioning in the computer involved providing inputs to the simulation that were based on the variety of studies showing that the CS and US are conveyed to the cerebellum by mossy fibers (Steinmetz et al., 1985; Lewis et al., 1987; Steinmetz et al., 1988) and climbing fibers (McCormick et al., 1985; Mauk et al., 1986), respectively (Fig. 1). The specific firing properties of these inputs were taken from published peristimulus histograms of CS and US activation of mossy (Aitkin and Boyd, 1978) and climbing (Sears and Steinmetz, 1991) fibers. Importantly, only a small number of the mossy fibers $(4 \%)$ were activated by the $\mathrm{CS}$, whereas the rest maintained their background activity during the CS. Thus, as is presumably the case in the real cerebellum, the simulation had to learn to respond to the CS, even when this stimulus engages only a small part of the total number of input fibers available. Finally, because evidence indicates that output of the cerebellum via the interpositus nucleus is necessary for the expression of the conditioned response (McCormick and Thompson, 1984; Krupa et al., 1993) and that during eyelid conditioning these nucleus neurons learn to increase their activity in response to the CS (McCormick and Thompson, 1984), the activity of the simulated nucleus cells was taken as a measure of the conditioned response (Fig. 1).

Animals. Data were obtained from 22 male New Zealand albino rabbits (Oryctolagus cuniculus), weighing 2.5-3.0 kg each (in addition, 28 rabbits were not included in the study because they showed no evidence for short-latency responses after picrotoxin infusion and histological examination revealed that the cannulas were misplaced). The animals were individually housed and had access to food and water ad libitum. Treatment of the animals and surgical procedures were in accordance with an approved animal welfare protocol.

Surgical preparation. All animals were first prepared with a cannula implanted in the anterior interpositus nucleus and with a head bolt cemented to the skull. Animals were preanesthetized with $5 \mathrm{mg} / \mathrm{kg}$ acepromazine, and their skulls were immobilized in a stereotaxic restrainer. Anesthesia was maintained with halothane (1-2\% mixed in oxygen), and sterile procedures were used during the placement of the cannulas. After exposing the skull, four holes were drilled to accommodate screws that would be used to affix a bolt to the skull. A large craniotomy was drilled just lateral to lambda and covered with bone wax. The head was positioned with lambda $1.5 \mathrm{~mm}$ ventral to bregma. A cannula (Plastics One, Roanoke, VA) consisting of a 26 gauge stainless steel guide sheath and a 33 gauge internal cannula that projected $1.2 \mathrm{~mm}$ beyond the tip of the guide sheath was placed at stereotaxic coordinates corresponding to the nucleus $(0.7 \mathrm{~mm}$ anterior, $4.9 \mathrm{~mm}$ left lateral, and $14.0 \mathrm{~mm}$ ventral to lambda). After placement, the electrode assembly and head bolt were secured to the skull with dental acrylic, and the skin was sutured. Two stainless steel stimulating electrodes were chronically implanted in the periorbital muscles rostral and caudal to the eye. Antibiotics, intravenous fluids, and analgesics were administered after surgery as needed, and animals were allowed at least 1 week to recover.

Conditioning procedures. The standard training session involved a Pavlovian conditioning delay protocol with a $500 \mathrm{msec}$ interstimulus interval. Each daily training session consisted of 12 nine trial blocks. Each block was comprised of eight paired presentations of the CS and US and one presentation of the CS only. The CS (a $1 \mathrm{kHz}, 85 \mathrm{~dB}$ tone) was presented for $550 \mathrm{msec}$ during CS-alone trials and coterminated with a $50 \mathrm{msec}$ train of constant current pulses (200 Hz, 1 msec pulse width, 2-3 mA) delivered to the periorbital electrodes during paired trials. Trials were separated by a random intertrial interval in the $25-35 \mathrm{sec}$ range. Stimulus presentation and data acquisition were controlled by a computer using custom software. Movement of the unrestrained eyelid was recorded by measuring the reflectance of an infrared light-emitting diode aimed at the eyelid. Voltage responses were determined to be linearly related to eyelid movement and were calibrated for each animal daily. 
Data analysis. Peak response amplitude, onset latency, and peak latency were calculated by custom software. Digitized sweeps (one point per msec) corresponded to the $200 \mathrm{msec}$ before and $2300 \mathrm{msec}$ after the CS onset. Once calibrated, peak amplitude was measured relative to an average of the $200 \mathrm{msec}$ baseline collected before CS onset. To be counted as a conditioned response, onset latency had to follow CS onset, and the movement amplitude had to reach $0.3 \mathrm{~mm}$ before US onset during paired trials. This criterion was relaxed for CS-alone trials in which movements were counted as conditioned responses if they reached a $0.3 \mathrm{~mm}$ amplitude at any time after CS onset. Trials in which there was $>0.3 \mathrm{~mm}$ of movement during the baseline were excluded from additional analysis. Onset latency was determined by calculating the point at which the response reached criterion.

Drug infusion. Test sessions with picrotoxin were conducted before acquisition, after $5 \mathrm{~d}$ of acquisition, after $5,15,30$, or $45 \mathrm{~d}$ of extinction, and after $5 \mathrm{~d}$ of reacquisition. All animals were tested after $5 \mathrm{~d}$ of acquisition and after $5 \mathrm{~d}$ of reacquisition but, to examine savings after different amounts of extinction, not all animals were tested at all of the extinction time points. The maximum and minimum numbers of test sessions received by a single animal were six and four, respectively. Except for the session given before acquisition, each test session began with four blocks of trials (CS plus US during acquisition or CS-alone during extinction) to establish a baseline of responding before drug inf usion. To minimize the effects of conditioning, the test session given before acquisition consisted of only $24 \mathrm{CS}$-alone trials. Then the session was paused, and $1 \mu \mathrm{l}$ of picrotoxin $(2 \mathrm{~mm}$ ) was infused at a rate of 0.5 $\mu \mathrm{l} / \mathrm{min}$. After waiting for $0.5 \mathrm{hr}$, the session was resumed and the animals received six more blocks of trials (CS plus US during acquisition or CS-alone during extinction). Animals were allowed at least $10 \mathrm{~d}$ between test sessions.

Histology. After training, the location of the lesion was determined for each animal using standard histological procedures. Briefly, the inf usion site was marked by passing a DC current (200 $\mu \mathrm{A}$ for $20 \mathrm{sec})$ through a small wire cut to the length of the internal cannula and exposed at the tip. Animals were killed with an overdose of sodium pentobarbital and perfused intracardially with 1.01 of $10 \%$ formalin. The brains were removed and stored in $10 \%$ formalin for several days. Brains were embedded in an albumin gelatin mixture, and the cerebellum was sectioned using a freezing microtome ( $80 \mu \mathrm{m}$ sections). Tissue was mounted, stained with cresyl violet, and counterstained with Prussian blue (for representative histological samples, see Fig. 7).

\section{RESULTS}

\section{Simulations show savings attributable to residual plasticity in the cerebellar nucleus}

The present experiments were designed to test mechanisms for savings suggested by a recently developed cerebellar simulation of eyelid conditioning (Medina et al., 2000). Building a detailed computer representation of the cerebellum was made possible by the extensive information available about cerebellar anatomy and physiology (Fig. 1; see Materials and Methods) (Eccles et al., 1967; Llinas, 1981; Ito, 1984; Voogd and Glickstein, 1998). Thus, the simulation is comprised of representations of cerebellar neurons and synapses whose connectivity and properties are derived from empirical findings. In addition, the simulation incorporated recent evidence for plasticity in both the cerebellar cortex ( $g r \rightarrow P k j$ synapses) and cerebellar nucleus ( $m f \rightarrow n u c$ synapses) (Robinson, 1976; Perrett et al., 1993; Raymond et al., 1996; Mauk, 1997). Based on empirical evidence, plasticity in the cerebellar cortex was controlled by climbing fiber inputs such that $g r \rightarrow P k j$ synapses active during a climbing fiber input decreased in strength (LTD) and those active in the absence of a climbing fiber input increased in strength (LTP) (Sakurai, 1987; Ito, 1989; Hirano, 1990; Shibuki and Okada, 1992; Linden, 1994; Salin et al., 1996). As first suggested by Miles and Lisberger (1981) and supported more recently by theoretical (Medina and Mauk, 1999) and empirical (Perrett and Mauk, 1995; Aizenman and Linden, 2000) analyses, plasticity in the cerebellar nucleus was controlled by inhibitory inputs from Purkinje cells. Thus, LTD of $m f \rightarrow n u c$ synapses occurred during strong inhibitory Purkinje cell inputs, whereas LTP was induced during transient pauses in this inhibition. The same results were obtained when plasticity at $m f \rightarrow n u c$ synapses was substituted by the recently reported changes in nucleus cell excitability, as long as this form of nonsynaptic plasticity remained under the control of Purkinje cell inputs (Aizenman and Linden, 2000). Eyelid conditioning was simulated by programming the computer to activate the simulated mossy fiber and climbing fiber afferents based on how these fibers are known to be activated by the CS (Aitkin and Boyd, 1978) and US (Sears and Steinmetz, 1991), respectively (Fig. 1; see Materials and Methods). Because eyelid conditioning has been shown to increase the activity of nucleus cells in response to the CS (McCormick and Thompson, 1984) and this increased activity is necessary for response expression (McCormick and Thompson, 1984; Krupa et al., 1993), the activity of the simulated nucleus cells during the CS was taken as a measure of learning (Fig. 1). Thus, our approach has been to build a simulation whose properties and parameters are constrained by what is known about cerebellar physiology and then to ask whether this simulation displays the capacity for eyelid conditioning. Neither the capability to acquire or extinguish conditioned responses nor the capability for savings were explicitly built into the simulation with hypothetical features or arbitrary parameters.

Although we have reported previously that this cerebellar simulation can acquire and extinguish conditioned responses (Medina et al., 2000), here we show that the simulation also displays robust savings during reacquisition (Fig. 2). As shown in the right panel of Figure $2 A$, simulated reacquisition was faster than original acquisition, which is shown with the gray line for comparison. Savings occurred in the simulations because of four factors. First, the acquisition of robust conditioned responses required the induction of plasticity in both the cerebellar cortex ( $g r \rightarrow P k j$ synapses) and cerebellar nucleus ( $m f \rightarrow n u c$ synapses), which is consistent with results from previous studies of cerebellar-mediated motor learning (Robinson, 1976; Perrett et al., 1993; Raymond et al., 1996; Mauk, 1997). Second, because of interactions between the rules for plasticity at these two sites, induction of plasticity in the cerebellar cortex and the ensuing change in simulated Purkinje cell activity was necessary to drive the induction of plasticity in the nucleus. These first two factors make the induction of plasticity at $m f \rightarrow n u c$ synapses the limiting factor for the rate of acquisition. Third, although the reversal of plasticity in the cerebellar cortex during extinction occurred quickly and was sufficient to suppress responding, plasticity in the nucleus reversed more slowly. Fourth, during simulated reacquisition, the presence of this residual plasticity in the cerebellar nucleus was responsible for the accelerated rate of relearning. Although our main goal is to test these predictions empirically, it is instructive to examine in more detail how the simulations produce savings.

In the simulations, residual plasticity in the cerebellar nucleus was produced by interactions between rules and sites of plasticity that made the induction of plasticity somewhat sequential. During simulated eyelid conditioning, the first changes involved decreased activity of Purkinje cells during the CS (Fig. 2B,C). This decrease was produced by the US activating the climbing fiber input and leading to the induction of LTD at CS-activated $g r \rightarrow P k j$ synapses as soon as acquisition training was started. In contrast, induction of plasticity at the $m f \rightarrow n u c$ synapses was not directly under the control of inputs activated by conditioning stimuli but was instead under the control of Purkinje cells (see 
Figure 2. Nucleus and Purkinje cell activity during simulated acquisition, extinction, and reacquisition. $A$, The changes in activity of simulated nucleus cells paralleled the changes in responding observed during eyelid conditioning. Nucleus cell activity increased during acquisition, decreased during extinction, and increased again during reacquisition at a faster rate (savings). The rate of original acquisition is shown in gray for comparison. $B$, The changes in nucleus activity shown in $A$ were produced by the induction of plasticity at $g r \rightarrow P k j$ synapses (shown as Cortex plasticity) and $m f \rightarrow n u c$ synapses (shown as $\mathrm{Nu}$ cleus plasticity). Because Purkinje cells are inhibitory whereas mossy fibers are excitatory, both LTD at $g r \rightarrow P k j$ synapses and LTP at $m f \rightarrow n u c$ synapses helped increase the activity of nucleus cells (during acquisition and reacquisition). Conversely, during extinction, LTP of $g r \rightarrow P k j$ synapses and LTD of $m f \rightarrow n u c$ synapses decreased nucleus cell activity. Note that, during extinction (middle), learning-induced plasticity at $g r \rightarrow P k j$ synapses was reversed rapidly, whereas reversal of plasticity at $m f \rightarrow n u c$ synapses took longer. This residual nucleus plasticity was responsible for savings in the simulations. $C$, Activity of a representative simulated Purkinje cell at different points during acquisition ( $A 0$, before acquisition; $A 1$, after 100 acquisition trials; $A 5$, after 500 acquisition trials) and extinction ( $E 1$, after 100 extinction trials; $E 5$, after 500 extinction trials). The long and short lines under the activity plots represent the CS and US, respectively. During acquisition, climbing fiber-induced LTD at $g r \rightarrow P k j$ synapses resulted in a reduction of Purkinje cell activity at approximately the time of the US $(A 1, A 5)$. In contrast, induction of LTP at these synapses restored the activity of Purkinje cells during extinction $(E 1, E 5)$. D, For the same time points as in $C$, the thin and thick lines represent, respectively, the average simulated nucleus cell activity during 50 trials before (Pre-infusion) and after (Picrotoxin) simulated disconnection of the cerebellar cortex. Initially during acquisition $(A 0, A 1)$, disconnection of the cerebellar cortex did not result in short-latency responses because LTP had not yet been induced at the $m f \rightarrow n u c$ synapses. However, by the end of simulated acquisition $(A 5)$, disconnection of the cerebellar cortex unmasked the presence of LTP at $m f \rightarrow n u c$ synapses by revealing short-latency responses. After extinction (E1), cerebellar cortex disconnection still unmasked these short-latency responses because $m f \rightarrow n u c$ synapses remained potentiated (see $B)$. If extinction was prolonged (E5), the magnitude of the short-latency responses began to decrease as $m f \rightarrow n u c$ synapses underwent LTD.

Materials and Methods). Therefore, during simulated conditioning, the induction of LTP at $m f \rightarrow n u c$ synapses could only begin after plasticity in the cerebellar cortex had started to produce transient decreases in Purkinje activity during the CS. As shown in Figure $2 B$, this necessarily makes the induction of plasticity at simulated $m f \rightarrow n u c$ synapses lag behind plasticity at $g r \rightarrow P k j$ synapses. Because the expression of robust responses in the simulations requires plasticity at both sets of synapses, the rate of initial acquisition was determined primarily by the delayed induction of LTP at the simulated $m f \rightarrow n u c$ synapses.

The changes that had been induced during acquisition were reversed in the same sequence during simulated extinction training, and this led to residual plasticity at the $m f \rightarrow n u c$ synapses after extinction. During simulated extinction, climbing fiber activity was inhibited when the CS was presented because of increased response-related inhibition coming from the cerebellar nucleus, as well as the decreased excitation from the absent US. The importance of nucleus cell inhibition of climbing fibers during simulated extinction is therefore consistent with findings that blockade of cerebellar nucleus output during extinction training prevents the extinction of conditioned responses (Ramnani and Yeo, 1996). The initial changes that resulted from the lack (inhibition) of climbing fiber activity during the CS were the induction of LTP at $g r \rightarrow P k j$ synapses, which restored the robust $\mathrm{CS}$ activity of the Purkinje cells (Fig. $2 B, C$ ). Indeed, as shown in the middle panel of Figure $2 B$, CS activity of Purkinje cells during simulated extinction was not simply reversed to its initial state but was instead slightly increased beyond it. This restored activity during the CS was sufficient to suppress responding and thus, consistent with a number of behavioral studies, the rate of extinction was significantly faster than the rate of acquisition (Schneiderman et al., 1962; Napier et al., 1992). In contrast to this rapid reversal of plasticity at $g r \rightarrow P k j$ synapses during extinction, the signals required to produce extinction-induced LTD at $m f \rightarrow n u c$ synapses did not occur until robust Purkinje cell activity had been restored. Thus, there was a prolonged period during extinction when conditioned responses were fully extinguished, but there remained residual plasticity in the nucleus at $m f \rightarrow n u c$ synapses (Fig. 2B, middle). During this period, no increases in nucleus cell activity were observed in response to the CS because the residual LTP at $m f \rightarrow n u c$ synapses was being counterbalanced by the slightly increased Purkinje cell activity that had resulted previously from the induction of LTP at $g r \rightarrow P k j$ synapses. Retraining at any time during this phase produced savings because the residual plasticity in the nucleus made the rate of relearning dependent only on the relatively quick induction of plasticity in the cortex.

This analysis leads to three testable predictions. First, during acquisition, the induction of plasticity in the nucleus should parallel the acquisition of behavioral responses, consistent with this plasticity being the initial limiting factor. Second, plasticity in the cerebellar nucleus should persist after conditioned responses have been extinguished. As shown in Figure 2D, the simulations revealed residual plasticity by the way in which short-latency 
responses could be unmasked after disconnection of the cerebellar cortex with simulated inf usion of picrotoxin in the cerebellar nucleus. In the simulations, these short-latency responses could be unmasked by picrotoxin even after conditioned responses had been fully extinguished. Third, the rate of reacquisition for individual rabbits should correlate with the magnitude of the short-latency responses unmasked on the last day of extinction before reacquisition. As a corollary of this last hypothesis, the simulations predict that, with extended extinction training, the amount of savings should be reduced as the $m f \rightarrow n u c$ synapses decrease in strength, and residual plasticity in the cerebellar nucleus is eventually eliminated (Fig. 2D, E5). As is always the case when modeling brain function, our simulations are only approximations. For example, residual plasticity in our simulations is a fundamental consequence of the sequential reversal of plasticity in the cerebellar cortex and nucleus during extinction. However, the precise rate at which nucleus plasticity is reversed during extinction and the exact duration of residual plasticity will depend on mechanistic details that are as yet unknown. Thus, although these predictions are specific, their scope is qualitative. The following sections present empirical tests of these three predictions.

\section{Prediction 1: rate of acquisition of short-latency responses}

The ability to test these predictions was facilitated by previous observations that the cerebellar cortex can be reversibly disconnected by blocking the downstream actions of Purkinje cells with inf usions of the GABA antagonist picrotoxin into the interpositus nucleus of the cerebellum (Garcia and Mauk, 1998). This study showed that infusing picrotoxin in the interpositus nucleus does not abolish conditioned responses completely but disrupts their learned timing. Normally conditioned responses are appropriately delayed to peak near the time the US occurs (Millenson et al., 1977; Mauk and Ruiz, 1992). After disconnection of the cerebellar cortex with picrotoxin infusion in the nucleus, responses display a short and relatively fixed latency to onset. In addition to blocking Purkinje cell input to the nucleus, the infusion of picrotoxin in the cerebellar nucleus is also likely to block the action of inhibitory interneurons. However, the emergence of short-latency responses after infusion of picrotoxin is probably caused mainly by the blockade of Purkinje cell input to the nucleus because lesions of the cerebellar cortex produce the same effects on response timing (Perrett et al., 1993; Perrett and Mauk, 1995; Garcia et al., 1999). Nevertheless, a contribution from inhibitory interneurons in generating short-latency responses cannot be ruled out. In the simulations, the short-latency responses were mediated by learning-induced plasticity in the interpositus nucleus. Although recent evidence is consistent with this view (Raymond et al., 1996; Garcia and Mauk, 1998; Nores et al., 1999; Steele et al., 1999; Aizenman and Linden, 2000), it is possible that extracerebellar sites of plasticity could play a role in generating the short-latency responses (see Discussion). However, it is the predicted residual nature of the plasticity mediating the shortlatency responses and not the localization of the site of plasticity that is essential for the results that follow regarding mechanisms for savings.

To examine the time course for induction of the plasticity responsible for the short-latency responses, we repeatedly disconnected the cerebellar cortex in the same group of rabbits throughout $5 \mathrm{~d}$ of training (Fig. 3; the number of rabbits participating in each average data point is given in parentheses under the hori-
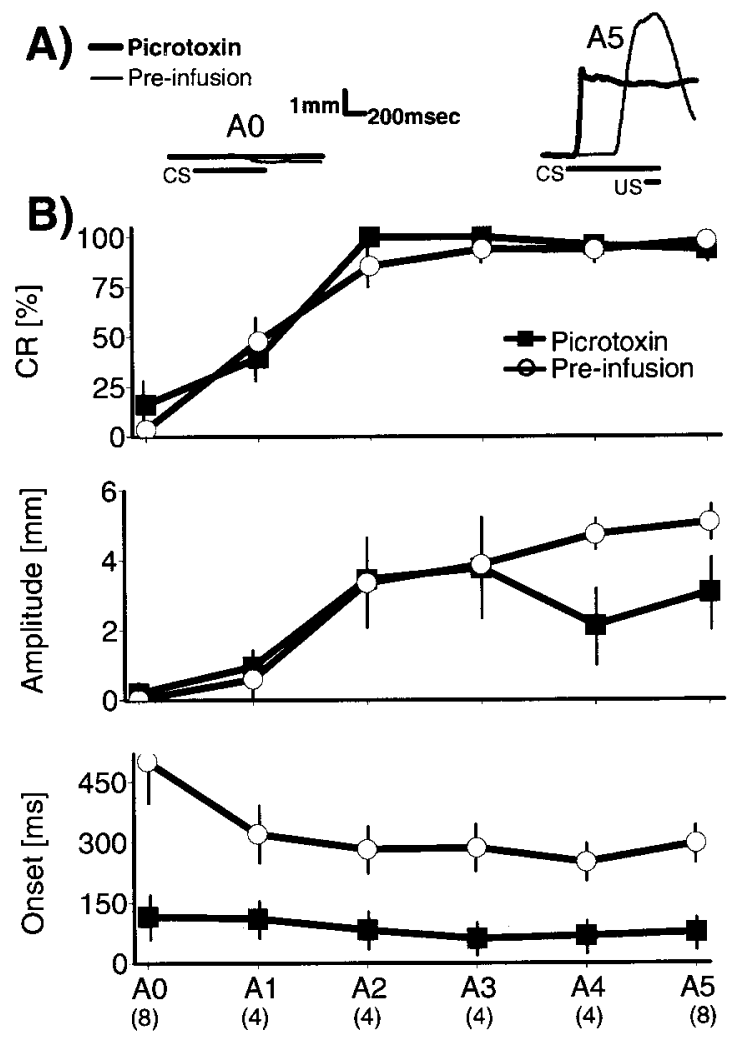

Figure 3. Comparison of conditioned responses assessed throughout acquisition training immediately before and after disconnection of the cerebellar cortex. $A$, Average conditioned responses for a representative animal on CS-alone trials before (thin line) and after (thick line) picrotoxin infusion into the interpositus nucleus. Before training $(A O)$, picrotoxin infusion did not unmask short-latency responses. After conditioned responses had been acquired during $5 \mathrm{~d}$ of training $(A 5)$, picrotoxin revealed short-latency responses. The long and short lines below the average sweeps correspond to times at which the CS and US were presented. $B$, Group data depicting the effects of acquisition on conditioned responding assessed before (open circles) and after (filled squares) picrotoxin infusion into the interpositus nucleus. Data before and after the infusion are from the same group of animals. Training resulted in parallel acquisition of timed (open circles) and short-latency (filled squares) responses, both in terms of frequency (top) and amplitude (middle). Responses measured after picrotoxin infusion exhibited a similar short-latency throughout acquisition (bottom, filled squares). The number of data points comprising each average is indicated in parentheses under the session label in the bottom.

zontal axis). Figure $3 A$ shows sample average eyelid traces in one representative rabbit (for histology, see Fig. 7) after picrotoxin inf usions before training was started and after $5 \mathrm{~d}$ of acquisition. In general, we observed that the rate of acquisition of shortlatency responses paralleled the acquisition of well timed behavioral responses observed in the same rabbits before the picrotoxin inf usion (Fig. 3B). During acquisition, the rate of increase in the frequency of short latency responding did not differ significantly from the rate of acquisition of well timed conditioned responses produced with an intact cerebellar cortex in the same rabbits. A two-way, mixed ANOVA performed to test for effects of picrotoxin and acquisition training on percentage conditioned responding over test sessions A0-A5 demonstrated significant effects for training $\left(F_{(5,28)}=42.16 ; p<0.001\right)$ but not for picrotoxin $\left(F_{(1,28)}=0.22\right)$, nor was there a significant interaction $\left(F_{(5,28)}=\right.$ $0.87)$. The small differences observed in the amplitudes of the responses in picrotoxin versus preinfusion for sessions A4 and A5 
were attributable to the rabbits squinting after the inf usion, which resulted in a partially closed eyelid throughout the infusion and a maximum amplitude of the short-latency response of $\sim 4 \mathrm{~mm}$. These results are consistent with the prediction that the induction of the plasticity mediating the short-latency response is a ratelimiting factor during initial acquisition, although they do not exclude contributions from other factors as well. Next, we examine the predictions related to savings and residual plasticity during extinction, which form the core of the present results.

\section{Prediction 2: residual plasticity after extinction}

To test for the presence of residual plasticity at the site mediating the short-latency responses, we began by repeatedly disconnecting the cerebellar cortex at different times during extinction in a second group of rabbits (Fig. 4; the number of rabbits participating in each average data point is given in parentheses under the horizontal axis). Although conditioned responses in the intact animals were in general completely extinguished by the end of the second day (Fig. 4B, top and middle, circles), infusion of picrotoxin into the interpositus nucleus of the same animals reliably unmasked short-latency responses after 5, 15, and $30 \mathrm{~d}$ of extinction (Fig. 4B, top and middle, squares). Indeed, short-latency responses were seen in two animals, even after $45 \mathrm{~d}$ of extinction training (for raw traces from these rabbits, see Figs. 4A, 5; for histology showing placement of cannulas, see Fig. 7). Whether these short-latency responses are mediated by plasticity in the interpositus nucleus of some other extracerebellar site, these results are consistent with the second prediction of the simulations; namely, that residual plasticity responsible for short-latency responses remains after conditioned responses have been fully extinguished.

Figure 4 also illustrates that, as predicted by the simulations, prolonged extinction eventually reverses residual plasticity as measured by a significant decrease in the amplitude and frequency of short-latency responses generated by picrotoxin infusion after $45 \mathrm{~d}$ of extinction $\left(F_{(4,38)}=49.01 ; p<0.001\right.$; Dunnett's test; $p<0.001)$. Importantly, the amplitude and frequency of short-latency responses recover with reacquisition training (Fig. $4 B$, black REACQ bar; no statistical difference between groups $A 5$ and $R E A C Q$ ), confirming that the decrease in short-latency responding observed during extinction is not a function of damage to the cerebellar circuitry or a consequence of picrotoxin losing effectiveness with repeated infusions.

\section{Prediction 3: the magnitude of residual plasticity correlates with the magnitude of savings}

To test this prediction, we compared the rate of reacquisition with the magnitude of the short-latency responses unmasked by picrotoxin the day before. Rabbits received 5, 15, or $45 \mathrm{~d}$ of extinction and were then retrained. As shown in Figure $6 A$, rabbits that showed strong evidence for residual plasticity on the last day of extinction before reacquisition (as indicated by the presence of big short-latency responses after infusion of picrotoxin) reacquired the conditioned response relatively quickly. In contrast, reacquisition was slower in rabbits that showed little evidence for residual plasticity the previous day. This relationship is revealed clearly in the five rabbits that were extinguished for $15 \mathrm{~d}$ (Fig. $6 \mathrm{~A}$, filled circles). Three of these rabbits reacquired the response very rapidly ( $\sim 35$ trials) after showing short-latency responses reliably on the previous day. The other two rabbits in this group showed little evidence for residual plasticity after $15 \mathrm{~d}$ of extinction and relearned the conditioned response at a much slower rate $(\sim 100$
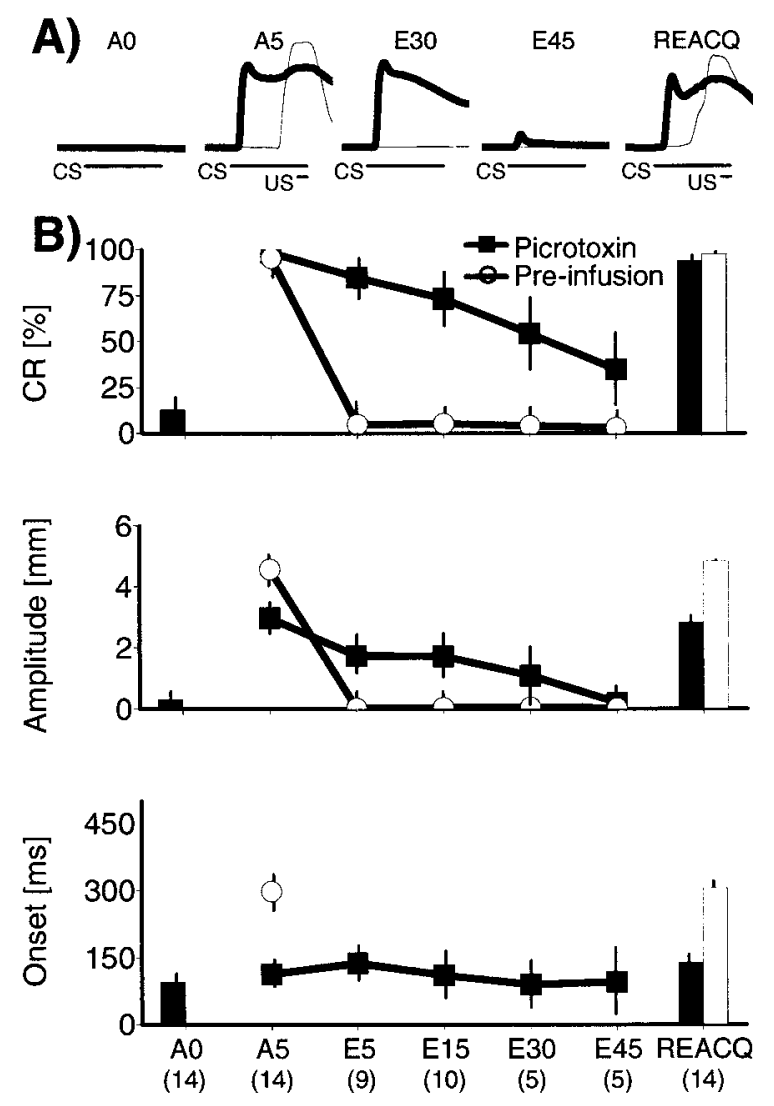

Figure 4. Comparison of conditioned responses assessed throughout extinction training immediately before and after disconnection of the cerebellar cortex. $A$, Average conditioned responses for a representative animal on CS-alone trials before (thin line) and after (thick line) picrotoxin infusion into the interpositus nucleus. Similar to the animal shown in Figure 3, this animal acquired short-latency and timed responses at similar rates (compare lines in $A O$ and $A 5$ ). In contrast, after timed responses had been fully extinguished (thin lines in E30 and E45), shortlatency responses could still be unmasked by picrotoxin infusions into the interpositus nucleus (thick lines in E30 and E45). B, Group data depicting the effects of extinction on conditioned responding assessed before (open circles and bars) and after ( filled squares and bars) picrotoxin inf usion into the interpositus nucleus. Data before and after the infusion are from the same group of animals (different animals from the data presented in Fig. 3 ). Extinction training decreased the frequency (top) and amplitude (middle) of both timed (open circles) and short-latency (filled squares) responding. The time course of extinction was much slower for shortlatency responses than for timed conditioned responses assessed before picrotoxin infusion. Robust short-latency responses could be consistently unmasked by picrotoxin infusions after complete extinction of conditioned responses $(E 5, E 15)$. With prolonged extinction, both the frequency and the amplitude of short-latency responses decreased (E30, E45). Importantly, the amplitude of short-latency responses recovered with reacquisition training $(R E A C Q)$, suggesting that the decreases in short-latency responding observed after prolonged extinction were not attributable to damage to the interpositus nucleus or picrotoxin losing effectiveness. Responses measured after picrotoxin infusion exhibited a similar short-latency onset throughout training and extinction (bottom). The number of data points comprising each average is indicated in parentheses under the session label in the bottom.

trials). Figure 7 shows sample cannula placements for some of these rabbits.

Surprisingly, the magnitude of the short-latency responses on the day before reacquisition was a better predictor of the rate of reacquisition than the total number of days of extinction training received before reacquisition. Although both the magnitude of the short-latency response $(p<0.001)$ and the days of extinction 

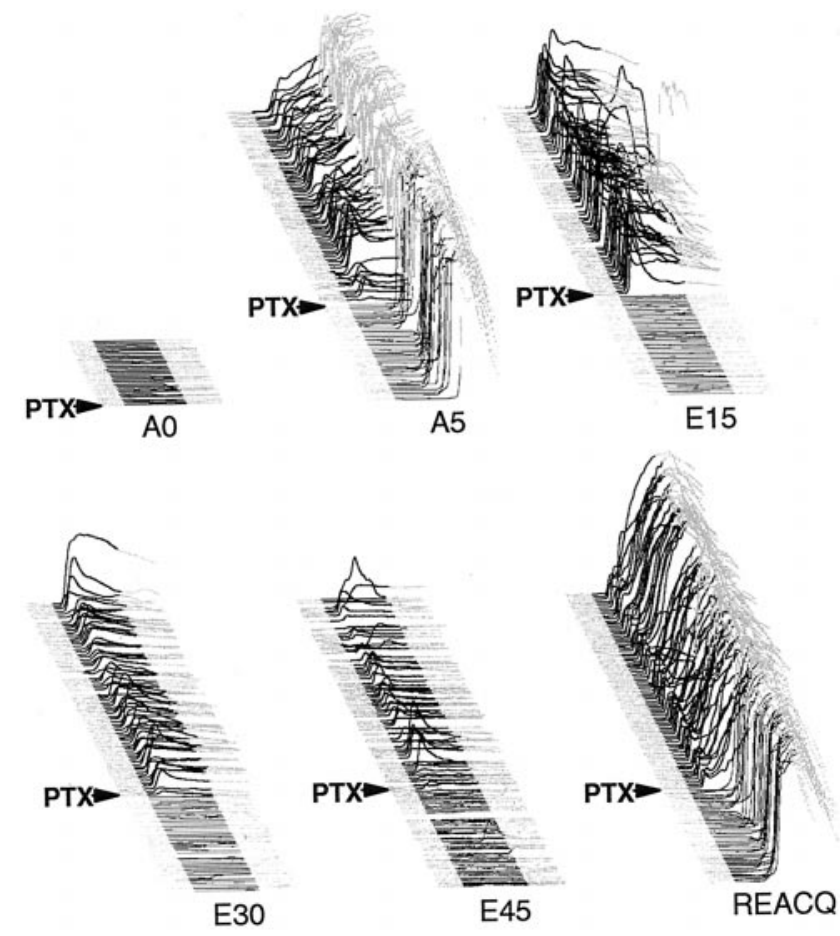

Figure 5. Raw eyelid traces throughout acquisition and extinction for one of the two animals that showed short-latency responses after $45 \mathrm{~d}$ of extinction. Each of the six plots shows all of the eyelid responses given during the training session before acquisition $(A O)$, at the end of $5 \mathrm{~d}$ of acquisition (A5), after 15 (E15), 30 (E30), or 45 (E45) d of extinction, and after reacquisition (REACQ). The location of the black arrow indicates the time during the session when picrotoxin $(P T X)$ was infused into the cerebellar nucleus. The black portion of each individual trace represents the time when the CS was being presented. Picrotoxin infusions unmasked short-latency responding to the CS long after conditioned responses had been extinguished.

$(p<0.05)$ were significantly correlated with the rate of reacquisition as shown in Figure $6 A$, correlation analysis showed that the magnitude of the short latency $(\mathrm{df}=12 ; r=-0.87)$ accounted for more of the variance seen in reacquisition than the total days of extinction ( $\mathrm{df}=12 ; r=0.64)$. The differences in correlation values between the two measures arose in part from the highly variable rates of reacquisition observed for the rabbits that were extinguished for $15 \mathrm{~d}$ (filled circles) and to the very rapid reacquisition of one rabbit that still showed strong evidence for residual plasticity even after $45 \mathrm{~d}$ of extinction (Fig. 6B, open square, bottom right corner). These results support the prediction from the simulations that the magnitude of residual plasticity before reacquisition is an important factor that contributes to the phenomenon of savings.

\section{DISCUSSION}

We have used eyelid conditioning in rabbits to test three predictions about cerebellar mechanisms for savings that were suggested by a large-scale computer simulation of the cerebellum. The simulations predicted that the slow rate of initial learning should parallel the induction of plasticity in the cerebellar nucleus, that extinction should only very slowly reverse this nucleus plasticity, and that, after extinction, savings should occur because residual plasticity in the nucleus contributes to the rapid rate of relearning. To test these three predictions, we made use of the ability to reversibly disconnect the cerebellar cortex during dif-
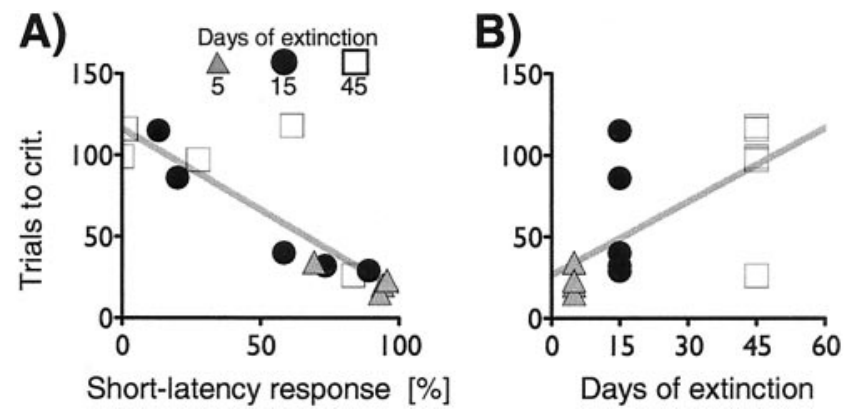

Figure 6. Two predictors of the rate of reacquisition. $A$, The scatterplot reveals a strong correlation between the magnitude of the short-latency response unmasked by picrotoxin on the last day of extinction and the amount of savings subsequently observed during reacquisition. Rabbits that were extinguished for 5,15 , or $45 \mathrm{~d}$ are shown as triangles, circles, and squares, respectively. Regardless of the amount of extinction that these rabbits had been exposed to, rabbits in which picrotoxin revealed consistent short-latency responses before reacquisition required fewer trials to relearn the conditioned response than rabbits in which picrotoxin did not unmask short-latency responses $(r=-0.87 ; p<0.001)$. $B$, The amount of extinction given before reacquisition was also correlated with the amount of savings observed during reacquisition $(r=0.64 ; p<0.05)$. Thus, in general, increasing the number of extinction sessions given before reacquisition training resulted in slower rates of reacquisition. However, this relationship was not as strong as in $A$, suggesting that residual plasticity, as measured by the ability of picrotoxin to unmask short-latency responses after extinction, is an even better predictor of the rate of reacquisition than the number of days of extinction training.
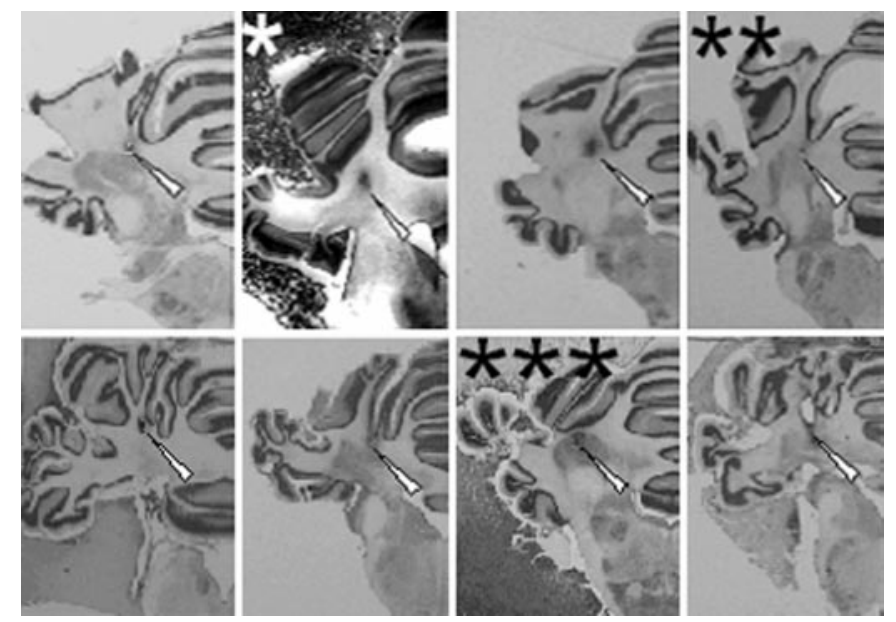

Figure 7. Examples of cannula placements. Sections showing a coronal view from eight of the 22 animals that were used in this study. The sections with *,**, or *** are from the rabbits whose raw data are shown in Figures $3 A, 4 A$, and 5 , respectively. The other five were chosen randomly from the remaining animals. The white arrows indicate the location of the cannula tip.

ferent phases of acquisition and extinction by infusing picrotoxin into the cerebellar nucleus of rabbits (Garcia and Mauk, 1998). This disconnection unmasks short-latency conditioned responses that are mediated by a site of plasticity outside of the cerebellar cortex, possibly in the cerebellar nucleus (Garcia and Mauk, 1998; Nores et al., 1999; Steele et al., 1999). Results from these reversible lesion experiments were consistent with all three of the predictions. First, the rate of acquisition of short-latency responses was similar to the rate of acquisition of normal conditioned responses. Second, the presence of residual plasticity was revealed by the ability of picrotoxin to unmask short-latency 
responses, even when normal conditioned responses had been extinguished for some time. Third, the rate of reacquisition was strongly correlated with the magnitude of the short-latency response unmasked during the last extinction session before reacquisition.

At some level of abstraction, the mechanism for savings suggested by our simulation is similar to the manner in which Kehoe's layered network model generates savings by protecting residual excitatory strength from the full consequences of extinction (Kehoe, 1988). However, unlike Kehoe's model, the present simulation predicted that, with extended extinction training, the amount of savings should be reduced as residual plasticity in the nucleus is slowly reversed. Although we have confirmed this prediction here, a previous study did not find any strong evidence to suggest that prolonged extinction training would slow down the rate of reacquisition (Napier et al., 1992). However, because the total amount of extinction given in the previous study was the equivalent of eight of our sessions (900 extinction trials), it is possible that reacquisition training was begun before cerebellar nucleus plasticity was significantly reversed. Indeed, the data presented in Figure 4 suggest that, on average, residual plasticity in the nucleus should be quite strong after eight sessions of extinction, which would account for the fast reacquisition rate observed in the previous study. Nevertheless, even in the results presented here, it was not possible to eliminate savings completely as revealed by the observation that, on average, reacquisition was still slightly faster than original acquisition, even after 45 sessions of extinction. Although our simulation predicts that once residual plasticity in the cerebellar nucleus has been completely reversed the rate of reacquisition should be indistinguishable from the rate of original acquisition, it is very likely that other factors (such as the animal becoming accustomed to the training environment) can also contribute to savings.

Excluding Kehoe's model (Kehoe, 1988), most hypothetical mechanisms for savings had centered previously around the proposition, first introduced by Pavlov (1927), that extinction cannot simply involve unlearning the original CS-US association and must instead require new (inhibitory) learning that opposes (but does not reverse) the learning accrued during acquisition (Scavio and Thompson, 1979; Klopf, 1988; Bouton, 1991). Indeed, the phenomenon of savings is among the evidence that has encouraged the general view that extinction cannot simply entail reversing the plasticity that was induced during acquisition. Although this may be true for many forms of learning, our results suggest how savings can occur, even with extinction mechanisms that reverse or unlearn changes that occurred during acquisition. As is the case in our simulations, savings may be explained by considering two sites of plasticity whose reversal during extinction occurs somewhat sequentially. Because cerebellar-mediated motor learning is inherently bidirectional, as shown not only during eyelid conditioning (Scavio and Thompson, 1979) but also during adaptation of the vestibulo-ocular reflex (VOR) (Miles and Eighmy, 1980) and saccades (Optican and Robinson, 1980), mutually reversing mechanisms of learning and extinction seem fitting and intuitive for the cerebellum. However, this may not be true for learning mediated by other brain systems.

Although the proposed mechanism for savings does not depend on the precise location of the plasticity mediating the shortlatency responses, recent evidence from behavioral and physiological studies is consistent with a site of plasticity in the cerebellar nucleus (Raymond et al., 1996; Garcia and Mauk, 1998; Nores et al., 1999; Steele et al., 1999; Aizenman and Linden, 2000). For example, we have found that the plasticity mediating the shortlatency responses can still be induced with training in which the downstream target of the cerebellar nucleus, the red nucleus, is blocked pharmacologically (Nores et al., 1999; Steele et al., 1999). Although these data do not distinguish between the contributions made by inhibitory (from Purkinje or interneurons) or excitatory (from mossy fibers) inputs to the nucleus, the results are inconsistent with the generation of short-latency responses by sites of plasticity downstream from the interpositus nucleus. Additional support for nucleus plasticity comes from studies of adaptation of the VOR. Using a combination of lesion, simulation, and detailed electrophysiological approaches, Lisberger and colleagues have found that the shortest latency changes in VOR modification involve plasticity at the vestibular nuclei (which are analogous to the deep cerebellar nuclei in eyelid conditioning) (Raymond et al., 1996). To the extent that comparisons between these two forms of cerebellar-dependent motor learning are valid, the VOR results suggest a role for plasticity in the cerebellar nuclei during eyelid conditioning (Raymond et al., 1996). Finally, the control of plasticity in the cerebellar nucleus by Purkinje cell inputs, which is key for the ability of the simulation to display savings, is supported by a recent examination of changes in the excitability of cerebellar nucleus cells (Aizenman and Linden, 2000). This study demonstrated that the excitability of cerebellar nucleus cells was increased after stimuli that resulted in $\mathrm{Ca}^{2+}$ entry. The authors argued that, in vivo, the $\mathrm{Ca}^{2+}$ transient that has been reported in response to a pause in Purkinje cell activity would suffice to induce the observed increase in excitability. The simulation results presented here were independent of changes in excitability versus synaptic strength, as long as these changes were controlled by Purkinje cell inputs. In principle, even de novo formation of new synapses in the cerebellar nuclei or plasticity at inhibitory interneurons, as long they are controlled by Purkinje cell inputs, are also consistent with both the results of the simulations and the reversible lesion data that we report here.

Arguments have been presented suggesting that a second site of plasticity outside the cerebellar cortex is not necessary for the expression of conditioned responses or at least that plasticity does not occur in the cerebellar nucleus (Yeo et al., 1985; Hesslow et al., 1999). Cerebellar nucleus plasticity is disputed by observations that lesions of the cerebellar cortex can abolish the expression of previously learned responses (Yeo et al., 1985). However, this observation is contradicted by recent studies, which have revealed short-latency responses after lesion or reversible disconnection of the cerebellar cortex and thus suggest that lesions that completely abolish conditioned responses may have involved inadvertent damage to the interpositus nucleus (Perrett et al., 1993; Perrett and Mauk, 1995; Garcia and Mauk, 1998; Garcia et al., 1999). Cerebellar nucleus plasticity mediated by the mossy fiber input has also been disputed on the basis that strong mossy fiber stimulation fails to elicit short-latency responses (Hesslow et al., 1999). It remains possible, however, that background Purkinje cell activity was able to suppress short-latency responses in this experiment and that evidence for short-latency responses would have been observed had the mossy fibers been stimulated during disconnection of the cerebellar cortex. In addition, this study does not rule out the possibility that plasticity in the cerebellar nucleus could be mediated by nonsynaptic changes. Thus, an important challenge for the future will be to determine with certainty the location of the plasticity that mediates the short-latency responses, to identify the nature of this plasticity (excitability, synaptic plasticity, and formation of new synapses), and to under- 
stand the signals that control its induction. For the present, our results suggest that, whatever the answers to these questions, residual plasticity mediating short-latency responses after the extinction of conditioned responses is at least partly responsible for the savings seen during relearning in eyelid conditioning.

\section{REFERENCES}

Aitkin LM, Boyd J (1978) Acoustic input to the lateral pontine nuclei. Hearing Res 1:67-77.

Aizenman CD, Linden DJ (2000) Rapid, synaptically driven increases in the intrinsic excitability of cerebellar deep nuclear neurons. Nat Neurosci 3:109-111.

Bouton ME (1991) Context and retrieval in extinction and in other examples of interference in simple associative learning. In: Current topics in animal learning: brain, emotion, and cognition (Dachowski L, Flaherty CF, eds), pp 25-53. Hillsdale, NJ: Erlbaum.

Chen C, Thompson RF (1995) Temporal specificity of long-term depression in parallel fiber-Purkinje synapses in rat cerebellar slice. Learn Mem 2:185-198.

Eccles JC, Ito M, Szentágothai J (1967) The cerebellum as a neuronal machine. Berlin: Springer.

Ekerot CF, Kano M (1989) Stimulation parameters influencing climbing fibre induced long-term depression of parallel fibre synapses. Neurosci Res 6:264-268.

Frey PW, Ross LE (1968) Classical conditioning of the rabbit eyelid response as a function of interstimulus interval. J Comp Physiol Psychol 65:246-250.

Garcia KS, Mauk MD (1998) Pharmacological analysis of cerebellar contributions to the timing and expression of conditioned eyelid responses. Neuropharmacology 37:471-480.

Garcia KS, Steele PM, Mauk MD (1999) Cerebellar cortex lesions prevent acquisition of conditioned eyelid responses. J Neurosci 19:10940-10947.

Hesslow G, Svensson P, Ivarsson M (1999) Learned movements elicited by direct stimulation of cerebellar mossy fiber afferents. Neuron 24:179-185.

Hirano T (1990) Depression and potentiation of the synaptic transmission between a granule cell and a Purkinje cell in rat cerebellar culture. Neurosci Lett 119:141-144.

Ito M (1984) The cerebellum and neural control. New York: Raven.

Ito M (1989) Long-term depression. Annu Rev Neurosci 12:85-102.

Karachot L, Kado RT, Ito M (1994) Stimulus parameters for induction of long-term depression in in vitro rat Purkinje cells. Neurosci Res 21:161-168.

Kehoe EJ (1988) A layered network model of associative learning: learning to learn and configuration. Psychol Rev 95:411-433.

Kim JJ, Thompson RF (1997) Cerebellar circuits and synaptic mechanisms involved in classical eyeblink conditioning. Trends Neurosci 20:177-181.

Klopf AH (1988) A neuronal model of classical conditioning. Psychobiology 16:85-125.

Krupa DJ, Thompson JK, Thompson RF (1993) Localization of a memory trace in the mammalian brain. Science 260:989-991.

Lewis JL, LoTurco JJ, Solomon PR (1987) Lesions of the middle cerebellar peduncle disrupt acquisition and retention of the rabbit's classically conditioned nictitating membrane response. Behav Neurosci 101:151-157.

Linden DJ (1994) Long-term synaptic depression in the mammalian brain. Neuron 12:457-472.

Llinas R (1981) Electrophysiology of the cerebellar networks. In: Handbook of physiology, Vol II, The nervous system (Brokks VB, ed), pp 831-976. Bethesda: American Physiological Society.

Mauk MD (1997) Roles of cerebellar cortex and nuclei in motor learning: contradictions or clues? Neuron 18:343-346.

Mauk MD, Ruiz BP (1992) Learning-dependent timing of Pavlovian eyelid responses: differential conditioning using multiple interstimulus intervals. Behav Neurosci 106:666-681.

Mauk MD, Steinmetz JE, Thompson RF (1986) Classical conditioning using stimulation of the inferior olive as the unconditioned stimulus. Proc Natl Acad Sci USA 83:5349-5353.

McCormick DA, Thompson RF (1984) Cerebellum: essential involvement in the classically conditioned eyelid response. Science 223:296-299.

McCormick DA, Steinmetz JE, Thompson RF (1985) Lesions of the inferior olivary complex cause extinction of the classically conditioned eyeblink response. Brain Res 359:120-130.

Medina JF, Mauk MD (1999) Simulations of cerebellar motor learning: computational analysis of plasticity at the mossy fiber to deep nucleus synapse. J Neurosci 19:7140-7151.

Medina JF, Garcia KS, Nores WL, Taylor NM, Mauk MD (2000) Timing mechanisms in the cerebellum: testing predictions of a large-scale computer simulation. J Neurosci 20:5516-5525.

Miles FA, Eighmy BB (1980) Long-term adaptive changes in primate vestibuloocular reflex. I. Behavioral observations. J Neurophysiol 43:1406-1425.

Miles FA, Lisberger SG (1981) Plasticity in the vestibulo-ocular reflex: a new hypothesis. Annu Rev Neurosci 4:273-299.

Millenson JR, Kehoe EJ, Gormezano I (1977) Classical conditioning of the rabbit's nictitating membrane response under fixed and mixed CS-US intervals. Learn Motiv 8:351-366.

Napier RM, Macrae M, Kehoe EJ (1992) Rapid requisition in conditioning of the rabbit's nictitating membrane response. J Exp Psychol Anim Behav Process 18:182-192.

Nores WL, Steele PM, Mauk MD (1999) Plasticity in the interpositus nucleus contributes to the expression of short-latency conditioned eyelid responses. Soc Neurosci Abstr 25:96.

Optican LM, Robinson DA (1980) Cerebellar-dependent adaptive control of primate saccadic system. J Neurophysiol 44:1058-1076.

Pavlov IP (1927) Conditioned reflexes: an investigation of the physiological activity of the cereb cortex. London: Oxford UP.

Perrett SP, Mauk MD (1995) Extinction of conditioned eyelid responses requires the anterior lobe of cerebellar cortex. J Neurosci 15:2074-2080

Perrett SP, Ruiz BP, Mauk MD (1993) Cerebellar cortex lesions disrupt learning-dependent timing of conditioned eyelid responses. J Neurosci 13:1708-1718.

Ramnani N, Yeo CH (1996) Reversible inactivations of the cerebellum prevent the extinction of conditioned nictitating membrane responses in rabbits. J Physiol (Lond) 495:159-168.

Raymond JL, Lisberger SG, Mauk MD (1996) The cerebellum: a neuronal learning machine? Science 272:1126-1131.

Reberg D (1972) Compound tests for excitation in early acquisition and after prolonged extinction of conditioned suppression. Learn Motiv $3: 246-258$

Robinson DA (1976) Adaptive gain control of vestibuloocular reflex by the cerebellum. J Neurophysiol 39:954-969.

Sakurai M (1987) Synaptic modification of parallel fibre-Purkinje cell transmission in in vitro guinea-pig cerebellar slices. J Physiol (Lond) 394:463-480.

Salin PA, Malenka RC, Nicoll RA (1996) Cyclic AMP mediates a presynaptic form of LTP at cerebellar parallel fiber synapses. Neuron 16:797-803.

Scavio MJJ, Thompson RF (1979) Extinction and reacquisition performance alternations of the conditioned nictitating membrane response. Bull Psyconom Soc 13:57-60.

Schactman T, Brown A, Miller R (1985) Reinstatement-induced recovery of a taste- $\mathrm{LiCl}$ association following extinction. Anim Learn Behav $13: 223-227$

Schneiderman N, Fuentes I, Gormezano I (1962) Acquisition and extinction of the classically conditioned eyelid response in the albino rabbit. Science 136:650-652.

Sears LL, Steinmetz JE (1991) Dorsal accessory inferior olive activity diminishes during acquisition of the rabbit classically conditioned eyelid response. Brain Res 545:114-122.

Shibuki K, Okada D (1992) Cerebellar long-term potentiation under suppressed postsynaptic $\mathrm{Ca}^{2+}$ activity. NeuroReport 3:231-234.

Steele PM, Nores WL, Medina JF, Mauk MD (1999) Induction of plasticity in the interpositus nucleus during eyelid conditioning requires input from the cerebellar cortex. Cold Spring Harb Symp Quant Biol Abstr 6:78.

Steinmetz JE, Lavond DG, Thompson RF (1985) Classical conditioning of the rabbit eyelid response with mossy fiber stimulation as the conditioned stimulus. Bull Psyconom Soc 28:245-248.

Steinmetz JE, Logan CG, Thompson RF (1988) Essential involvement of mossy fibers in projecting the conditioned stimulus to the cerebellum during classical conditioning. In: Cellular mechanisms of conditioning and behavioral plasticity (Woody DL, Alkon DL, McGaugh JL, eds), pp 143-148. New York: Plenum.

Thompson RF (1986) The neurobiology of learning and memory. Science 233:941-947.

Thompson RF, Krupa DJ (1994) Organization of memory traces in the mammalian brain. Annu Rev Neurosci 17:519-549.

Voogd J, Glickstein M (1998) The anatomy of the cerebellum. Trends Neurosci 21:370-375.

Yeo CH, Hardiman MJ, Glickstein M (1985) Classical conditioning of the nictitating membrane response of the rabbit. II. Lesions of the cerebellar cortex. Exp Brain Res 60:99-113. 